\title{
Upper extremity deep vein thrombosis after in vitro fertilization treated with ultrasound accelerated catheter directed thrombolysis
}

\author{
Mert Dumantepe, ${ }^{1}$ Osman Fazlıoğullar,,${ }^{1}$ Mustafa Seren, ${ }^{2}$ İbrahim Uyar ${ }^{3}$ \\ Received: October 10, 2014 Accepted: November 27, 2014 Published online: February 10, 2015
}

\begin{abstract}
Upper extremity deep vein thrombosis (UEDVT) is an important clinical entity, presenting with an increased risk for developing fatal pulmonary thromboembolism. A 32-year-old woman was admitted to our hospital complaining swelling and pain of the right upper extremity for five days. The patient was diagnosed as primary infertility and underwent in vitro fertilization after stimulation with gonadotropin-releasing hormone analog one month prior to her admission. An ultrasound scan showed thrombosis of the right brachiocephalic and subclavian veins with partial obstruction of the lumen. The patient was successfully treated with EkoSonic ${ }^{\circledR} \mathrm{Mach} 4 \mathrm{e}$ Endovascular device with an overnight infusion of recombinant human tissue plasminogen activator. Catheter-directed thrombolysis may represent a potential therapeutic alternative for patients with severe UEDVT, particularly in those in whom medical management fails or conventional thrombolytic therapy and surgical thrombectomy are contraindicated or considered extremely risky.
\end{abstract}

Keywords: Catheter directed thrombolysis; deep vein thrombosis; in vitro fertilization; ultrasound.

Upper extremity deep venous thrombosis (UEDVT) is a serious disease, which is based on the pathogenesis and in view of the individual patient's prognosis and must be divided into primary and secondary presentations. ${ }^{[1]}$ Primary UEDVT is a rather benign disease with an excellent prognosis, having only a minor potential of developing disabling postthrombotic syndrome (PTS). Secondary UEDVT, on the other hand, typically occurs in older patients with severe comorbidities, mainly related to indwelling central venous catheters and cancer. It may also present during pregnancy or as a complication of assisted reproductive techniques. As a consequence of the underlying diseases, prognosis of secondary UEDVT remains poor. ${ }^{[2]}$

Regardless of the etiology, the cornerstone of therapy is anticoagulant treatment with low molecular weight heparin or unfractionated heparin and vitamin $\mathrm{K}$ antagonists to prevent thrombus progression and pulmonary embolism. Due to the lack of evidences, the optimal duration of treatment still remains unclear. ${ }^{[2,3]}$

Recent advances in catheter-based interventions have led to the development of a variety of minimally invasive endovascular strategies to remove venous thrombus.

Ultrasound accelerated catheter-directed thrombolysis (UACDT) has been developed to resolve the existing thrombus in a rapid and complete manner.
This technique integrates high frequency, low intensity ultrasound (US) with standard CDT to accelerate clot dissolution, reducing treatment time as well as the incidence of thrombolysis-related complications. ${ }^{[4,5]} \mathrm{It}$ is hypothesized that catheter-directed thrombolysis for UEDVT provides an effective solution with less severe PTS than during traditional anticoagulation. Herein, we report a case of UEDVT following in vitro fertilization (IVF) who was successfully treated with UACDT.

\section{CASE REPORT}

A 32-year-old woman was admitted to our hospital with complaints of swelling and painful right upper extremity during the preceding five days. The patient was previously diagnosed with primary infertility and underwent IVF after stimulation with a gonadotropinreleasing hormone analogue one month prior to her admission. On admission, the patient described a tight, painful swelling of her face, upper chest, and arms. The patient history also revealed slight swelling

1Department of Cardiovascular Surgery, Medical Park Gebze Hospital, Kocaeli, Turkey ${ }^{2}$ Department of Cardiovascular Surgery, Dışkapı Ylldırım Beyazıt Training and Research Hospital, Ankara, Turkey

${ }^{3}$ Akut Cardiovascular Hospital, İzmir, Turkey

Corresponding author: Mert Dumantepe, M.D. Medical Park Gebze Hastanesi Kalp ve Damar Cerrahisi Bölümü, 41420 Gebze, Kocaeli, Turkey.

Tel: +90 532 - 3771872 e-mail: mdumantepe@gmail.com 
of the neck and face lasting for a month, mainly noticed during morning hours, which resolved later during the day. On clinical examination, her body temperature was $37.2{ }^{\circ} \mathrm{C}$, pulse was 78 beats $/ \mathrm{min}$, respiratory rate was 18 breaths $/ \mathrm{min}$, blood pressure was $116 / 70 \mathrm{mmHg}$, and oxygen saturation was $94 \%$ on room air. She was plethoric with deep swelling of her face, neck and right arm. Her heart sounds were normal and abdomen was soft and non-tender without any evidence of mass or organomegaly. Complete blood count and biochemistry results were also normal. Chest X-ray produced unremarkable findings with no evidence of pneumothorax, consolidation, tracheal deviation, pleural effusion, or mediastinal widening. An ultrasound scan showed thrombosis of brachiocephalic and subclavian veins with partial obstruction of the lumen (Figure 1a).

The patient underwent UACDT using the EKOS EkoSonic Endovascular System (EKOS Corporation, Bothell, WA), which consisted of a $5.2 \mathrm{~F}, 106 \mathrm{~cm}$ long infusion catheter, an ultrasonic core wire, and a control unit with catheter interface cables. We preferred a $30 \mathrm{~cm}$ length treatment zone catheter in our case, which was appropriate for the thrombus length. The drug delivery catheter was navigated over a 0.035 -inch guide wire, therefore, the treatment zone traversed the entire clot and the tip exited the thrombus. After final positioning, the guide wire was exchanged for a matching ultrasonic core wire containing a series of US transducer elements $(2.2 \mathrm{MHz}, 0.45 \mathrm{~W})$ distributed $1.0 \mathrm{~cm}$ apart to evenly deliver US energy radially along the distal coaxial infusion zone (Figure 1b). After priming the drug lumens of the catheter with sub-therapeutic heparin $(1,000 \mathrm{U} / \mathrm{mL})$, continuous infusion of thrombolytic agent was initiated through the side-holes of the infusion delivery catheter using Alteplase (Actilyse, Boehringer Ingelheim $\mathrm{GmbH}$, Germany), a recombinant human tissue plasminogen activator, at a $5 \mathrm{mg}$ bolus, followed by an ultrasound accelerated infusion at $0.02 \mathrm{mg} / \mathrm{kg} / \mathrm{h}$ over 14 hours. Normal saline, which included $1 \mathrm{mg}$ Alteplase, was infused via a coolant port connected to the central lumen (and discharging from the distal end) at a rate of $35 \mathrm{~mL} / \mathrm{hr}$ to dissipate any small amount of heat-generated by the US energy. The energy was delivered via the core wire with simultaneous infusion of the thrombolytic drug. Fibrinogen and activated partial thromboplastin time (aPTT) levels were taken every six hours during thrombolytic infusion.

The patient was monitored at the intensive care unit and post-procedure venography was performed prior to removing the introducer. Venography showed excellent flow in the subclavian and brachiocephalic vein (Figure 1c). There were no bleeding complications due to the treatment. The patient was discharged on the second postoperative day on vitamin $\mathrm{K}$ antagonist therapy for six months. The follow-up US scan showed normalized subclavian vein and upper extremity veins (Figure 2). Her symptoms improved immediately after the UACDT and she continues to be symptom-free a week after the procedure.

\section{DISCUSSION}

Compared to lower extremity DVT, UEDVT is an uncommon disease representing about $10 \%$ of all DVTs. ${ }^{[1]}$ It can be associated with mechanical or anatomical factors (e.g. insertion of central venous
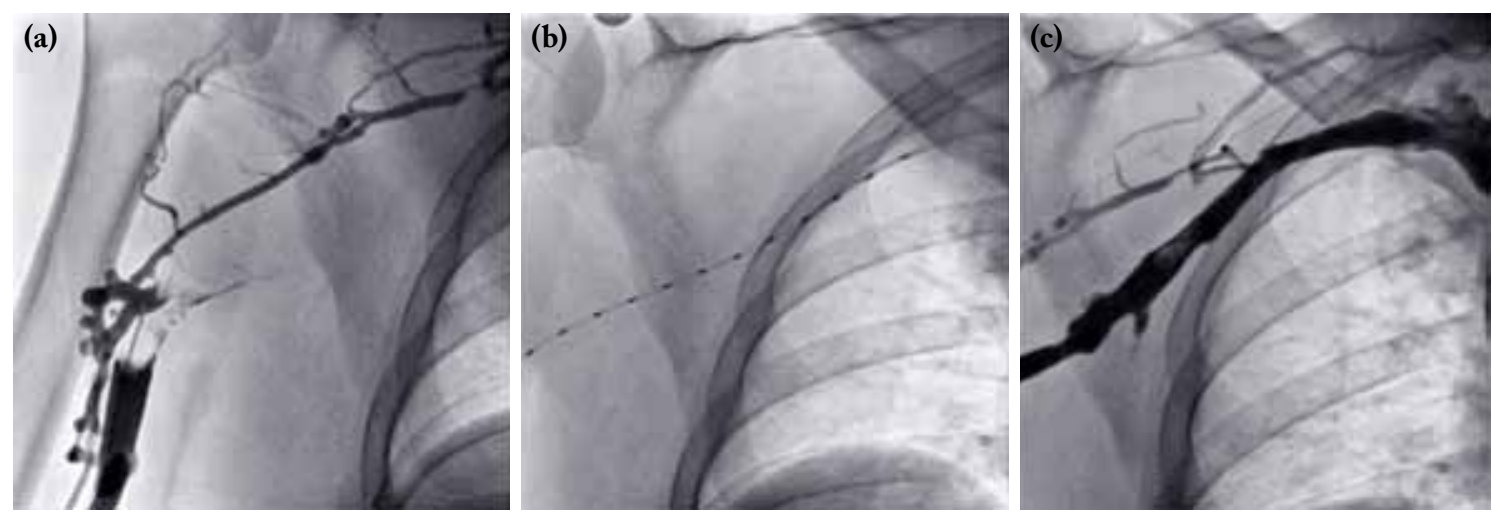

Figure 1. (a) Venography showing the absence of filling of the subclavian and brachiocephalic vein, consistent with thrombosis. (b) EKOS catheter with a $30 \mathrm{~cm}$ treatment length was placed into the thrombus (note the radiopaque ultrasound transducers). (c) Final venogram showing complete resolution. 

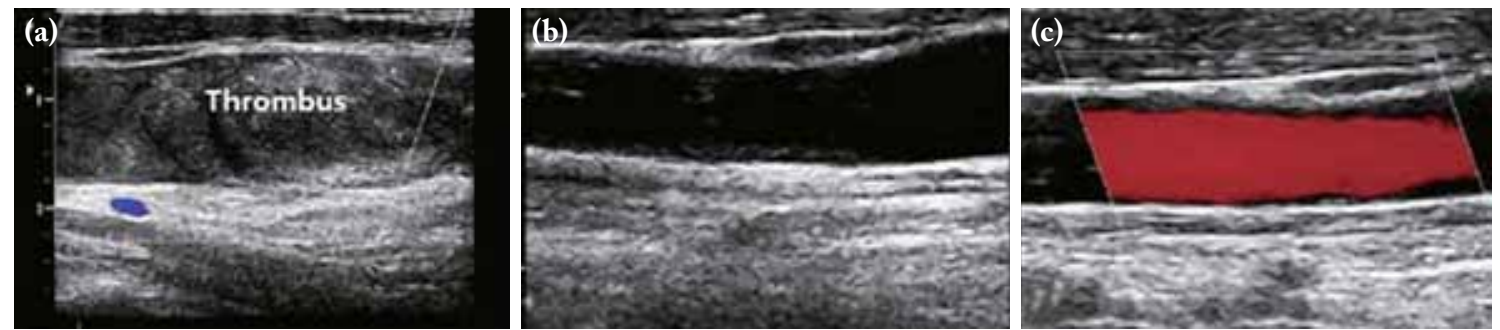

Figure 2. (a) The longitudinal image of the right neck shows thrombosis of the right subclavian vein with obstruction of the lumen. $(\mathbf{b}, \mathbf{c})$ The follow-up ultrasound scan reveals normalized subclavian vein.

catheters or narrowing of veins by cervical rib) and hypercoagulable states (e.g. anti-thrombin deficiency, malignancy and anti-phospholipid syndromes). ${ }^{[2]}$ In addition, UEDVT can be associated with pulmonary embolism (PE) with reported incidence rates of about up to one third of all patients. ${ }^{[3]}$ Since our patient's vital signs were normal and there was little clinical evidence of $\mathrm{PE}$, the possibility of $\mathrm{PE}$ was considered to be low. Moreover, echocardiographic examination showed normal right ventricular function and normal pulmonary arterial pressure.

It has been assumed that thrombolytic therapy for UEDVT may reduce the risk of long-term complications, especially the troubling PTS, characterized by chronic arm and hand aching and swelling due to early restoration of venous patency, thereby minimizing damage to vessel valves and endothelium. Recent retrospective $\mathrm{e}^{[6]}$ and prospective $\mathrm{e}^{[7,8]}$ cohort studies of conventional anticoagulant therapy for unselected UEDVT reported to be $27 \%$ to $44 \%$ mild PTS and almost no severe PTS. Although some cohort studies have evaluated short-term efficacy, adverse events, and reocclusion rates, we found no reports assessing the development of PTS after initial UACDT with subsequent conventional anticoagulant therapy for UEDVT.

Due to its rarity and possible variations in anatomical obstruction associated with UEDVT, there is no unique and unequivocal management strategy. Most patients are unresponsive to anticoagulation alone, which appears to be effective only in the mildest cases. However, life-long anticoagulation following definitive endovascular therapy may be important to reduce the incidence of reocclusion and may play a role in maintaining collateral circulation. ${ }^{[9]}$

Endovascular stents have become the treatment of choice for acute symptom relief of such patients. When stent placement is difficult or dangerous due to the presence of a large amount of thrombus, thrombectomy, or thrombolysis should be attempted prior to stent deployment. On the other hand, stent placement for treatment of UEDVT is not devoid of complications. Infections, pulmonary embolus, stent migration, hematoma at the insertion site, bleeding, and rarely, perforation or rupture of the venous structures resulting in death, have been previously reported. ${ }^{[10]}$

As thrombus is deemed to be the cause of the upper extremity deep venous obstruction, removing it using UACDT with a low dose of thrombolytic agent may result in reduced risk of hematoma, gastrointestinal bleeding, and shorter length of hospital stay with all the relevant cost and comorbidities. To overcome the limitations posed by long-term treatment and highdrug doses in catheter directed thrombolysis, UACDT enhances drug permeation through thrombus by disaggregating the fibrin matrix, exposing additional plasminogen receptor sites to the thrombolytic agent. ${ }^{[4,5]}$ The US energy affects thrombus in the entire venous segment, increasing the probability of complete thrombus clearance. The significant benefit of complete thrombolysis, compared to partial lysis, has been demonstrated in previous studies of standard thrombolysis. ${ }^{[11]}$ Therefore, the ability of this technique to penetrate and resolve the entire thrombus may potentially lead to increased long-term patency rates and better long-term outcomes.

Recent literature data have shown a potential benefit of adjuvant angioplasty and stent implantation of the residual venous stenosis in patients with UEDVT. ${ }^{[12]}$ Also, Alteplase has a high-degree of safety with few complications when delivered by a catheter rather than the traditional systemic infusion.

In conclusion, UACDT may represent a potential therapeutic alternative in patients with severe UEDVT, particularly in those in whom medical management 
fails or conventional thrombolytic therapy and surgical thrombectomy are contraindicated or considered extremely risky.

\section{Declaration of conflicting interests}

The authors declared no conflicts of interest with respect to the authorship and/or publication of this article.

\section{Funding}

The authors received no financial support for the research and/or authorship of this article.

\section{REFERENCES}

1. Bernardi E, Pesavento R, Prandoni P. Upper extremity deep venous thrombosis. Semin Thromb Hemost 2006;32:729-36.

2. Chan WS, Ginsberg JS. A review of upper extremity deep vein thrombosis in pregnancy: unmasking the 'ART' behind the clot. J Thromb Haemost 2006;4:1673-7.

3. Prandoni P, Polistena P, Bernardi E, Cogo A, Casara D, Verlato F, et al. Upper-extremity deep vein thrombosis. Risk factors, diagnosis, and complications. Arch Intern Med 1997; 157:57-62.

4. Dumantepe M, Tarhan A, Yurdakul I, Özler A. US-accelerated catheter-directed thrombolysis for the treatment of deep venous thrombosis. Diagn Interv Radiol 2013;19:251-8.

5. Grommes J, Strijkers R, Greiner A, Mahnken AH, Wittens $\mathrm{CH}$. Safety and feasibility of ultrasound-accelerated catheter-directed thrombolysis in deep vein thrombosis. Eur J Vasc Endovasc Surg 2011;41:526-32.

6. Prandoni P, Bernardi E, Marchiori A, Lensing AW, Prins $\mathrm{MH}$, Villalta $\mathrm{S}$, et al. The long term clinical course of acute deep vein thrombosis of the arm: prospective cohort study. BMJ 2004;329:484-5.

7. Persson LM, Arnhjort T, Lärfars G, Rosfors S. Hemodynamic and morphologic evaluation of sequelae of primary upper extremity deep venous thromboses treated with anticoagulation. J Vasc Surg 2006;43:1230-5.

8. Kahn SR, Elman EA, Bornais C, Blostein M, Wells PS. Postthrombotic syndrome, functional disability and quality of life after upper extremity deep venous thrombosis in adults. Thromb Haemost 2005;93:499-502.

9. Dumantepe M, Tarhan A, Ozler A. Successful treatment of central venous catheter induced superior vena cava syndrome with ultrasound accelerated catheter-directed thrombolysis. Catheter Cardiovasc Interv 2013;81:E269-73.

10. Lanciego C, Chacón JL, Julián A, Andrade J, López L, Martinez B, et al. Stenting as first option for endovascular treatment of malignant superior vena cava syndrome. AJR Am J Roentgenol 2001;177:585-93.

11. Mewissen MW, Seabrook GR, Meissner MH, Cynamon J, Labropoulos N, Haughton SH. Catheter-directed thrombolysis for lower extremity deep venous thrombosis: report of a national multicenter registry. Radiology 1999;211:39-49.

12. Delis KT, Bjarnason H, Wennberg PW, Rooke TW, Gloviczki P. Successful iliac vein and inferior vena cava stenting ameliorates venous claudication and improves venous outflow, calf muscle pump function, and clinical status in post-thrombotic syndrome. Ann Surg 2007;245:130-9. 